\title{
A NEW METHOD FOR THE MEASUREMENT OF MAXIMAL FAT OXIDATION: A PILOT STUDY
}

\author{
Pritt Purge ${ }^{1}$, Pritt Lehismets ${ }^{2}$, JaAk JÜRImÄE ${ }^{1}$ \\ ${ }^{1}$ Institute of Sport Pedagogy and Coaching Sciences, University of Tartu, Estonia \\ ${ }^{2}$ Centre of Behavioral and Health Sciences, University of Tartu, Estonia
}

\begin{abstract}
Exercise intensity is one of the main factors determining the rate of fat oxidation during exercise. The 3-min incremental protocol is desirable for practical reasons and allows for the estimation of fat oxidation across a wide range of exercise intensities. However, the use of a small number of exercise intensities traditionally used to estimate fat oxidation does not allow for the precise estimation of exercise intensity at which the rate of fat oxidation is maximal (Fat ${ }_{\max }$ ). The purpose of this study was to examine the validity of the determination of Fat max $_{\text {adopting 1-min }}$ step incremental bicycle ergometer protocol. In this study we also compared $\mathrm{Fat}_{\max }$ determined by 3-min step incremental bicycle ergometer test with Fat ${ }_{\text {max }}$ measured by 1-min step icremental bicycle ergometer test. The average peak oxygen consumption $\left(\mathrm{VO}_{2 \text { peak }}\right)$ for the entire group of subjects was $48.85 \pm 7.58 \mathrm{ml} / \mathrm{min} / \mathrm{kg}$. The Fat max $_{\text {occurred }}$ at $47.2 \pm 4.9 \%$ of $\mathrm{VO}_{2 \text { peak }}$ in Test 1 and at $51.3 \pm 7.4 \%$ of $\mathrm{VO}_{2 \text { peak }}$ in Test 2, which corresponded to $35.8 \pm 7.4 \%$ and $36.1 \pm 8.0 \%$ of the maximal workload ( $\mathrm{W}_{\max }$ ) in Tests 1 and 2, respectively. Heart rate (HR) in Fat max $_{\text {was }}$ at $63.9 \pm 6.8 \%$ and $62.1 \pm 6.2 \%$ from maximal HR in Tests 1 and 2, respectively. There were no significant differences $(\mathrm{p}<0.05)$ between work rate $(\mathrm{WR})$, respiratory coefficent (RER) and HR at Fat ${ }_{\text {max }}$ during both tests. Similary, the values of Fat $\mathrm{max}_{\max }$ and $\mathrm{VO}_{\text {fatmax }}$ were not significantly different in both tests. It appeared that the proposed 1-min step incemental exercise protocol can be used for the measurement of Fat $t_{\max }$ in physically active males as the obtained results were not different from previously used methods to calculate Fat ${ }_{\text {max }}$. However, the Fat ${ }_{\max }$ results are different as fat oxidation rate is affected by endurance, sex, age, body composition, exercise type and exercise duration.
\end{abstract}

Keywords: soccer, fat oxidation, aerobic capacity, Fat ${ }_{\max }$ 


\section{INTRODUCTION}

It is known that exercise intensity is one of the main factors determining the rate of fat oxidation during exercise [2]. Absolute fat oxidation rates increase during low-to-moderate exercise intensities and then markedly decline at high intensities, implying that there is an exercise intensity at which the rate of fat oxidation is maximal (Fat ${ }_{\max }$ ) [15]. The highest rate of fat oxidation during submaximal exercise has been reported to occur between 40 and $65 \%$ of peak oxygen consumption $\left(\mathrm{VO}_{2 \text { peak }}\right)$ [1]. Exercise intensity that promotes the maximum fat oxidation rate has been termed as Fat ${ }_{\max }$ and is typically expressed as a percentage of $\mathrm{VO}_{2 \text { peak }}$ [14]. It has been suggested that training at this intensity may have utility for endurance performance [2] and body mass loss [3]. In addition, it has been suggested that high fat oxidation rates can be beneficial for a large variety of individuals [2].

Most studies have only investigated three or four different intensties to

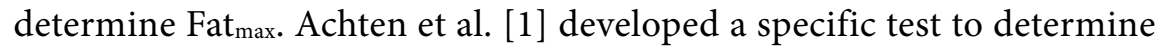
the exercise intensity which elicits maximal fat oxidation (MFO). This Fat $_{\max }$ consisted considered of a graded exercise test to exaustion on a bicycle ergometer, starting at $95 \mathrm{~W}$ with $35 \mathrm{~W}$ increments every 3 min during which gas exchange measurements were performed. Using this protocol, Fat ${ }_{\text {max }}$ occured at $64 \% \mathrm{VO}_{2 \text { peak }}$ [2]. The 3-min incremental protocol is desirable for practical reasons and allows for the estimation of fat oxidation rate across a wide range of exercise intensities. Consequently, this protocol has been adapted for other studies in adults [16]. However, the use of a relatively small number of exercise intensities traditionally used to estimate fat oxidation does not allow for the precise estimation of Fat max $_{\text {[14]. }}$ [14

A number of studies have used a progressive exercise test lasting 8-12 min to determine $\mathrm{VO}_{\text {2peak }}$ when assessing Fat ${ }_{\max }[8,12]$, while others have determined both fat oxidation and $\mathrm{VO}_{2 \text { peak }}$ during the same test lasting around $30 \mathrm{~min}$ and longer [14]. Bircher et al. [4] reported that the protocol that was more time-consuming indicated a higher Fat max $_{\text {ax }}$ and MFO than those done by a shorter protocol, although the increments for both protocols were small. These data indicated that Fat max $_{\max }$ may be influenced by exercise duration, probably due to changes in substrate concentrations and hormonal responses [15]. In contrast, Achten et al. [1] concluded that exercise duration did not affect Fat max $_{\max }$ determination. It is possible that the use of an incremental protocol to determine both $\mathrm{MFO}$ and $\mathrm{VO}_{2 \text { peak }}$ does not provide a valid measure of $\mathrm{VO}_{2 \text { peak }}$ as lower $\mathrm{VO}_{\text {peak }}$ values were observed from protocols lasting 20-28 min compared with 8-12 $\mathrm{min}$ in adults [17]. 
The purpose of this pilot stady was to examine the validity of the determination of Fat $t_{\max }$ adopting 1-min step incremental bicycle ergometer protocol. In this study we compared Fat ${ }_{\text {max }}$ during traditional 3-min step incremental bicycle ergometer test with Fat ${ }_{\max }$ measured by 1-min step icremental bicycle ergometer test. It was hypothesised that Fat $t_{\max }$ determined at both protocols are similar but a fat oxidation rate is higher using longer 3-min step protocol.

\section{MATERIALS AND METHODS}

\section{Participants}

Thirteen soccer players aged 18-30 years volunteered to participate in this study. They were recruited from local training groups and had a training history of $4.5 \pm 1.5$ years and had trained regularly for minimal 3 times per week for at least last two years. None of the participants was receiving any medications or had any disease. All procedures were approved by the Medical Ethics Committee of the University of Tartu.

\section{General design}

All subjects performed two graded exercise tests to exhaustion on a bicycle ergometer (Lode, Groningen, The Nederland). Test 2 was performed one week after Test 1 . The results of these tests were used to determine the exercise intensity that elicits maximal fat oxidation.

\section{Anthropometry and body composition assessment}

Body height $(\mathrm{cm})$ was measured with the use of a Martin metal anthropometer to the nearest $0.1 \mathrm{~cm}$ according to the standard technique, and body mass was measured with minimal clothing to the nearest $0.05 \mathrm{~kg}$ with a medical electronic scale (A\&D Instruments, Abingdon, UK). BMI was calculated as body mass $(\mathrm{kg})$ divided by body height $\left(\mathrm{m}^{2}\right)$. Dual-energy X-ray absorptiometry (DXA) scans of the total body were performed using a QDR Discovery scanner (Hologic, Waltham, MA, USA). Total body scans were analyzed for body fat mass (FM) and fat free mass (FFM) values using Hologic APEX software version 3.3.0.1.

\section{Experimental design}

The Test 1 was performed on an electronically braked bicycle ergometer (Lode Corival, Netherlands). Parcipants performed an initial work rate at 
$60 \mathrm{~W}$ with an increments of $20 \mathrm{~W}$ after every $1 \mathrm{~min}$. Parcipants cycled at a cadence of $70 \pm 5 \mathrm{rpm}$, and they were actively encouraged to continue until volutional exhausion. Heart rate was recorded every $5 \mathrm{~s}$ during the test using a commercially available HR monitor (Polar RS400, Polar Electro, Kempele, Finland). Gas exchange variables were measured throughout the test in a breath-by-breth mode and data were stored in $10 \mathrm{~s}$ intervals. Oxygen consumption $\left(\mathrm{VO}_{2}\right)$, carbon dioxide output $\left(\mathrm{VCO}_{2}\right)$ and minute ventilation $\left(\mathrm{V}_{\mathrm{E}}\right)$ were continuously measured using portable open spirometry system (MetaMax 3B, Cortex, Leipzig, Germany). The analyser was calibrated with gases of known concentration before each test according to the manufacturer's guidelines. All data were calculated by means of computer analysis using standard software (MetaMax-Analysis 3.21, Cortex, Leipzig, Germany). Peak oxygen consumption was achieved when two of the following three criteria were fullfilled: 1) $\mathrm{VO}_{2}$ plateau defined as a failure of $\mathrm{VO}_{2}$ to increase by greater than $2.0 \mathrm{ml} / \mathrm{min} / \mathrm{kg}$ with increase of test load; 2) $\mathrm{HR} \geq 95 \%$ from the predicted individual maximum (formula 220-age); and/or 3) $R E R \geq 1.05$.

Test 2 was performed one week after Test 1 on an electronically braked cycle ergometer (Lode Corival, Netherlands). Parcipants performed an initial work rate at $95 \mathrm{~W}$ with an increments of $35 \mathrm{~W}$ after every $3 \mathrm{~min}$. Both position on the bike and cadence was kept similar during both trials. Heart rate was recorded every $5 \mathrm{~s}$ during the test using a commercially available HR monitor (Polar RS400, Polar Electro, Kempele, Finland). Gas exchange variables were measured throughout the test in a breath-by-breth mode and data were stored in $10 \mathrm{~s}$ intervals. Oxygen consumption, $\mathrm{VCO}_{2}$ and $\mathrm{V}_{\mathrm{E}}$ were continuously measured using portable open spirometry system (MetaMax 3B, Cortex, Leipzig, Germany). The analyser was calibrated with gases of known concentration before each test according to the manufacturer's guidelines.

All data were calculated by means of computer analysis using standard software (MetaMax-Analysis 3.21, Cortex, Leipzig, Germany). Aerobic threshold (AeT), anaerobic threshold (AnT), $\mathrm{VO}_{2 \text { peak }}, \mathrm{V}_{\mathrm{E}}$, and maximal work rate $\left(W R_{\max }\right.$ ) were calculated using MetaMax-Analysis 3.21 software (Cortex, Leipzig, Germany) in each subject.

Fat oxidation rates were determined from $\mathrm{VO}_{2}$ and $\mathrm{VCO}_{2}$ values averaged over the last minute of each stage using the following equation [6]:

Fat oxidation $=1.67 \times \mathrm{VO}_{2}-1.67 \times \mathrm{VCO}_{2}$

Carbohydrite oxidation $=4.55 \times \mathrm{VCO}_{2}-3.21 \times \mathrm{VO}_{2}$

Fat oxidation rates were calculated for all stages in which $\mathrm{RER}<1.00$. The exercise intensity (W) associated with the highest recorded fat oxidation rate was selected as Fat $\max$. 


\section{Statistical analysis}

Statistical analysis was performed with SPSS 17.0 for Windows (Chicago, IL, USA). Means and \pm SD were determined. Evaluation of normality was performed with the Shapiro-Wilk statistical method.. The difference of the performance data between the two incremental cycling tests and Fatmax were tested with a paired sample t-test. The level of significance was set at $\mathrm{p}<0.05$.

\section{RESULTS}

The average $\mathrm{VO}_{2 \text { peak }}$ for the entire group was $48.85 \pm 7.58 \mathrm{ml} / \mathrm{min} / \mathrm{kg}$ (Table 1). Figure 1 shows the relationship between fat oxidation rate and exercise intensity expressed in $\mathrm{W}$ at every workstage. With increasing exercise intensities the fat oxidation rate increased to $31.78 \pm 10.46 \mathrm{~g} / \mathrm{h}$ in Test 1 and to $37.15 \pm 7.16 \mathrm{~g} / \mathrm{h}$ in Test 2 at $121.54 \pm 25.12 \mathrm{~W}$ in Test 1 and $121.92 \pm 29.12 \mathrm{~W}$ in Test 2 beyond which the oxidation rate decreased.

Table 1. Anthropometrical and functional parameters of the subjects.

\begin{tabular}{lccc}
\hline $\mathbf{n}=\mathbf{1 3}$ & $\mathbf{X} \pm \mathrm{SD}$ & Minimum & Maximum \\
\hline Age $(\mathrm{yrs})$ & $23.77 \pm 2.74$ & 21.0 & 30.0 \\
\hline Height $(\mathrm{cm})$ & $184.54 \pm 7.20$ & 175.0 & 198.0 \\
\hline Weight $(\mathrm{kg})$ & $85.82 \pm 12.05$ & 69.2 & 111.9 \\
\hline $\mathrm{AeT}(\mathrm{l} / \mathrm{min})$ & $139.92 \pm 6.85$ & 127.0 & 152.0 \\
\hline AeT $(\mathrm{W})$ & $186.72 \pm 21.75$ & 159.7 & 233.3 \\
\hline AnT $(\mathrm{l} / \mathrm{min})$ & $170.62 \pm 6.95$ & 157.0 & 181.0 \\
\hline AnT $(\mathrm{W})$ & $275.96 \pm 27.65$ & 225.0 & 336.0 \\
\hline $\mathrm{VO}_{2 \max }(\mathrm{kg} / \mathrm{min} / \mathrm{kg})$ & $48.85 \pm 7.58$ & 34.0 & 58.0 \\
\hline $\mathrm{VO}_{2 \max }(\mathrm{l} / \mathrm{min})$ & $4.13 \pm 0.49$ & 3.4 & 5.0 \\
\hline $\mathrm{VO}_{2 \max }(\mathrm{W})$ & $338.90 \pm 33.99$ & 280.3 & 383.0 \\
\hline $\mathrm{V}_{\mathrm{E}}(\mathrm{L} / \mathrm{min})$ & $149.19 \pm 26.27$ & 105.4 & 189.6 \\
\hline $\mathrm{WR}_{\max }(\mathrm{W})$ & $351.62 \pm 26.73$ & 313.3 & 388.7 \\
\hline $\mathrm{WR}_{\max }(\mathrm{W} / \mathrm{kg})$ & $4.17 \pm 0.66$ & 2.8 & 5.0 \\
\hline $\mathrm{HR}_{\max }(\mathrm{l} / \mathrm{min})$ & $190.85 \pm 7.15$ & 179.0 & 203.0 \\
\hline Fat mass $(\mathrm{kg})$ & $15.88 \pm 3.97$ & 11.4 & 24.4 \\
\hline Body fat $\%$ & $18.73 \pm 2.72$ & 14.1 & 24.6 \\
\hline Lean body mass $(\mathrm{kg})$ & $68.17 \pm 8.76$ & 56.4 & 88.3 \\
\hline
\end{tabular}

AeT - aerobic threshold; $\mathrm{AnT}$ - anaerobic threshold; $\mathrm{VO}_{2 \max / \mathrm{kg}}$ - maximal oxygen concuption scale to body mass; $\mathrm{VO}_{2 \max }$ - maximal oxygen concuption; $\mathrm{V}_{\mathrm{E}}-$ maximal ventilation; $\mathrm{WR}_{\max }-$ maximal work rate; $\mathrm{HR}_{\max }$ - maximal heart rate. 

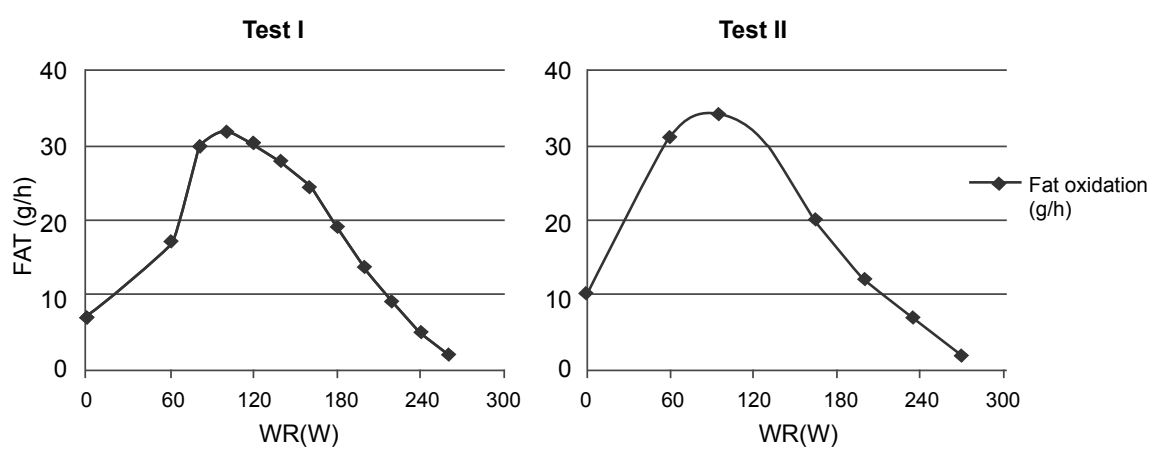

Figure 1. Fat oxidation in two different incremental workload tests.

The Fat ${ }_{\text {max }}$ occurred at 47.2 $\pm 4.9 \% \mathrm{VO}_{2 \text { peak }}$ in Test 1 and at $51.3 \pm 7.4 \% \mathrm{VO}_{2 \text { peak }}$ in Test 2, which corresponds to $35.8 \pm 7.4 \%$ ad $36.1 \pm 8.0 \%$ of $\mathrm{WR}_{\max }$ in

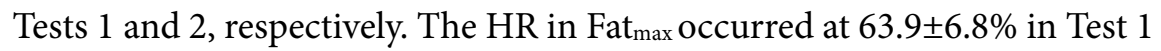
and in $62.1 \pm 6.2 \%$ in Test 2 from $\mathrm{HR}_{\max }$ (Figure 2).

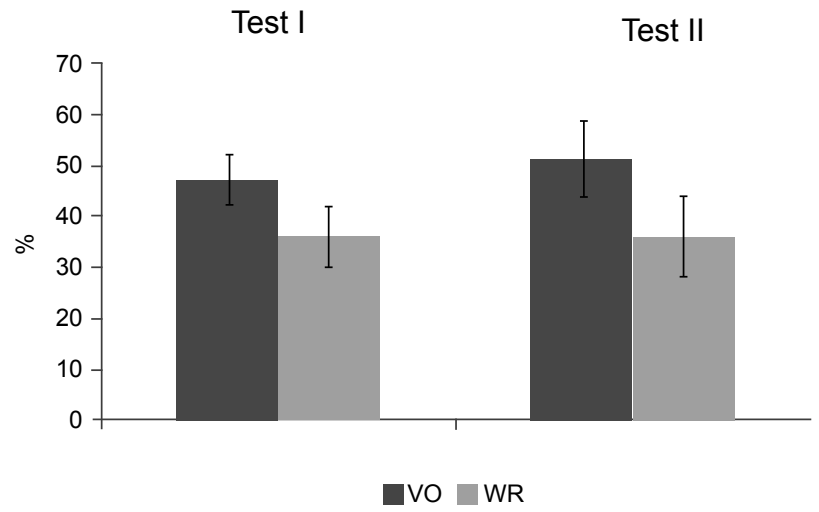

Figure 2. Fat max $_{\text {max }}$ at $\mathrm{VO}_{\text {peak }} \%$ and $\mathrm{WR}_{\max } \%$ in two different workload protocol in healthy active men.

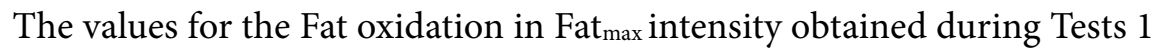
and 2 in bicycle ergometer are presented in Table 2 . There were no differences $(\mathrm{p}<0.05)$ between WR, RER and HR during both tests. Similary, the values of $\mathrm{MFO}$ and $\mathrm{VO}_{2}$ compared in both tests were not signifficant diferent, however, the values were higher in Test 2 than in Test 1. 
Table 2. Results of Fat max $_{\text {in }}$ two different test methods.

\begin{tabular}{lccc}
\hline $\mathbf{n = 1 3}$ & Test I & Test II & $\mathbf{p}$ \\
\hline $\mathrm{MFO}(\mathrm{g} / \mathrm{h})$ & $31.78 \pm 10.46$ & $37.15 \pm 7.16$ & 0.08 \\
\hline $\mathrm{CHO}(\mathrm{g} / \mathrm{h})$ & $69.89 \pm 24.17$ & $68.64 \pm 32.90$ & 0.87 \\
\hline $\mathrm{VO}_{2}(\mathrm{~L} / \mathrm{min})$ & $1.95 \pm 0.29$ & $2.11 \pm 0.37$ & 0.08 \\
\hline $\mathrm{VCO}_{2}(\mathrm{~L} / \mathrm{min})$ & $1.63 \pm 0.24$ & $1.74 \pm 0.37$ & 0.16 \\
\hline $\mathrm{RER}$ & $0.84 \pm 0.05$ & $0.82 \pm 0.04$ & 0.23 \\
\hline $\mathrm{WR}(\mathrm{W})$ & $121.54 \pm 25.12$ & $121.92 \pm 29.12$ & 0.95 \\
\hline $\mathrm{HR}(\mathrm{I} / \mathrm{min})$ & $122.00 \pm 14.54$ & $118.77 \pm 14.21$ & 0.30 \\
\hline
\end{tabular}

MFO- maximal fat oxidation; CHO-maximal carbohydrate oxidation; VO- oxygen consumption; VCO- carbon dioxide consumption; WR- work rate; HR- heart rate

\section{DISCUSSION}

The aim of the present study was to investigate whether it is possible to measure Fat $t_{\max }$ in healthy active males with 1-min long stage incremental exercise protocol in bicycle and compare the results with the suggested 3-min long stages incremental exercise protocol [1]. The first test allows to measure in same time fat oxidation parameters and determine $\mathrm{VO}_{2}$ peak. We found no differences $(\mathrm{p}>0.05)$ in Fat ${ }_{\max }$ results in both tests and in this case it is possible to measure Fat max $_{\text {in }}$ healty active males using the suggested shorter 1-min incremental exercise protocol.

Fat oxidation during exercise has typically been assessed using a small number of prolonged stady state exercise bouts to ensure the valid use of indirect calometry in adults $[7,13]$. Several studies have measured fat oxidation in different populations generally using 3-6 min long incremental exercise potocols [14]. Achten et al. [1] developed and validated a protocol to identify Fat max $_{\text {ax }}$ using a 3-min long and wide range of exercise intensities in trained adult males. Fat oxidation was estimated from the final $2 \mathrm{~min}$ of each 3 -min stage of the incremental exercise test [1]. It was concluded that an incremental exercise test on a bicycle ergometer starting at $95 \mathrm{~W}$ with $35 \mathrm{~W}$ increments every 3-min to exhausion can be used for the determination of $\mathrm{Fat}_{\max }$ and the rate of MFO in trained adult males [14]. A further issue surrounding the use of incremental protocols is that fat oxidation during the later exercise stages may be influenced by the earlier stages, i.e. a residual or carry-over effect [5], although Achten et al. [1] reported that previous stages had no influence on measured fat oxidation. 
The studies that are available indicate that Fat max $_{\max }$ generally occurs between 30 and $60 \%$ of $\mathrm{VO}_{2 \text { peak }}$ in young people [14]. Previous studies with healthy active males have found that Fat $\mathrm{m}_{\max }$ occurred at $64 \% \mathrm{VO}_{2 \text { peak }}$ [2] and $61-64 \% \mathrm{VO}_{2 \text { peak }}$ [10]. In our study the $\mathrm{Fat}_{\max }$ was $47.16 \pm 4.88 \%$ and $51.31 \pm 7.37 \%$ of $\mathrm{VO}_{2 \text { peak }}$ in Tests 1 and 2, respectively. In the second test, the used $35 \mathrm{~W}$ incremental step was considered to be high to determine exacly the Fat ${ }_{\max }$ intensity [2]. Accordingly, it can be suggested that the use of lower stage $(20 \mathrm{~W})$ increases the number of stages participants are able to complete and increase the precision with which Fat max $_{\text {is }}$ estimated. Achten et al. [1] reported that with lower incremental stage the test will be more longer. However, they found no differences in two different workload protocols and suggested to use $35 \mathrm{~W}$ stage increments.

While in our first test we used $20 \mathrm{~W}$ with 1-min stage increments, the test duration was not longer as usually, however, th participants did not rech stady-state at 1-min stage time [9], which can affect the results. The reason why Achten et al. [1] had higher Fat max $_{\text {max }}$ results compared to our study was because the parcipants in Achten et al. [1] study were endurance-trained cyclists and runners with higher $\mathrm{VO}_{2 \text { peak values. Although when compared }}$ our results with results in Achten and Jeukendrup [2] and Lima-Silva et al. [10] studies, there were also higher results in maximal fat oxidation. In our study, maximal fat oxidation was $0.53 \pm 0.17 \mathrm{~g} / \mathrm{min}$ and $0.62 \pm 0.12 \mathrm{~g} / \mathrm{min}$ in Test 1 and 2, respectively. In Achten et al. [1] study maximal fat oxidation was $0.60 \pm 0.07 \mathrm{~g} / \mathrm{min}$. Achten and Jeukendrup [2] investigated endurancetrained athletes and found similar fat oxidation results $(0.52 \pm 0.15 \mathrm{~g} / \mathrm{min})$ as in our first test. Many authors have found that maximal fat oxidation is higher in endurance-trained parcipants [10,11], hovewer, the results of our study are similar to other studies [2,3] or even lower [10].

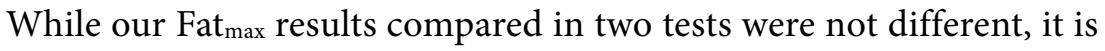
possible that the suggested exercise test protocol with 1-min stages can be used to evaluate Fat $t_{\max }$ paramateres and also to determine $\mathrm{VO}_{2 \text { peak. }}$. It was probably possible because the physical activity level in our parcipants was relative high, although the same test could not be suitable for measure Fat ${ }_{\max }$ in overweight or physically inactive people.

It was concluded that the proposed 1-min step incemental exercise protocol can be used to measure Fat $t_{\max }$ in physically active males, because the results between two tests were not significantly different. 


\section{REFERENCES}

1. Achten J, Gleeson M, Jeukendrup AE. (2002) Determination of the exercise intensity that elicits maximal Fat oxidation. Med Sci Sports Exerc, 34: 92-97

2. Achten J, Jeukendrup AE. (2003) Maximal Fat oxidation during exercise in trained men. Int J Sports Med, 24: 603-608

3. Achten J, Jeukendrup AE. (2004) Relation between plasma lactate concentration and fat oxidation rates over a wide range of exercise intensities. Int J Sports Med, 25: 32-37

4. Bircher S, Knechtle B, Müller G, Knecht H. (2005) Is the highest fat oxidation rate coincident with the anaerobic threshold in obese women and men? Eur J Sport Sci, 5: 79-87

5. Brooks GA, Mercier J. (1994) Balance of carbohydrate and lipid utilization during exercise: The "crossover" concept. J Appl Physiol, 76: 2253-2261

6. Frayn KN. (1983) Calculation of substrate oxidation rates in vivo from gaseous exchange. J Appl Physiol, 55, 628-634

7. Friedlander AL, Casazza GA, Homing MA, Buddinger TF, Brooks GA. (1998) Effects of exercise intensity and training on lipid metabolism in young women. Am J Physiol, 276: 853-863

8. Froelicher VF, Jr Brammell H, Davis G, Noguera I, Stewart A, Lancaster MC. (1974) A comparison of three maximal treadmill exercise protocols. J Appl Physiol, 36, 720-725

9. Gaessar GA, Poole DC. (1996) The slow component of oxygen uptake kinetics in humans. Exerc Sport Sci Rev, 24: 35-71

10. Lima-Silva AE, Bertuzzi RC, Pires FO, Gagliardi JF, Barros RV, Hammond J, Kiss MA. (2010) Relationship between training status and maximal Fat oxidation rate. J Sports Sci Med, 9: 31-35

11. Nordby P, Saltin B, Helge JW. (2006) Whole-body Fat oxidation determined by graded exercise and indirect calorimetry: a role for muscle oxidative capacity? Scand J Med Sci Sports, 16: 209-214

12. Pollock ML, Foster C, Schmidt D, Hellman C, Linnerud AC, Ward A. (1982) Comparative analysis of physiologic responses to three different maximal graded exercise test protocols in healthy women. Am Heart J, 103: 363-373

13. Romijn JA, Coyle EF, Hibbert, J, Wolfe RR. (1992) Comparison of indirect calorimetry and a new breath $13 \mathrm{C} / 12 \mathrm{C}$ ratio method during strenuous exercise. Am J Physiol, 1992; 263: 64-71.

14. Zakrzewski JK, Tolfrey K. (2011) Fatmax in children and adolescents: A review. Eur J Sport Sci, 11: 1-18

15. Takagi S, Sakamoto S, Midorikawa T, Konishi M, Katsumura T. (2014) Determination of the exercise intensity that elicits maximal fat oxidation in short-time testing. J Sport Sci, 32: 175-182 
16. Venables CV, Achten J, Jeukendrup AE. (2005) Determinants of Fat oxidation during exercise in healthy men and women: a cross-sectional study. J Appl Physiol, 98: 160-167

17. Weston SB, Gray AB, Schneider DA, Gass GC. (2002) Effect of ramp slope on ventilation thresholds and VO2peak in male cyclists. Int J Sports Med, 23: $22-27$

\section{Correspondence to:}

Priit Purge, $\mathrm{PhD}$

Institute of Sport Pedagogy and Coaching Sciences

University of Tartu

Jakobi 5, 51014, Tartu, Estonia

Telephone: +3727375372

E-mail: priit.purge@ut.ee 